\title{
2021 AADSM Virtual Annual Meeting Abstracts
}

Disclaimer: The following are the abstracts and case reports accepted for the 2021 Virtual Annual Meeting.

Abstracts and case reports do not follow the same peer-review process followed for the submission of original research articles for the Journal of Dental Sleep Medicine. Rather, all submissions were blind peer-reviewed for acceptance by members of the AADSM Scientific Committee. The committee uses criteria to score research abstracts which include (but are not limited to) applicability to dental sleep medicine, novelty, clarity, proper research methodology and data analysis, well-founded conclusions and creativity. Criteria to score case reports include (but are not limited to) applicability, uniqueness, clarity, well-founded discussion and creativity.

It is important to keep in mind that abstracts and case reports presented at the Annual Meeting are intended to spur education and discussion for both attendees and authors.

Brand names are not permitted to be used in titles and are limited to two references within the submission body. Furthermore, the abstracts include author disclosures of any conflicts of interest or affiliation with a company. If a company or governmental body provided any financial support for the research, this is also disclosed. The AADSM does not endorse or recommend any products or services presented in these abstracts.

ABSTRACT \#001

\section{EFFECTS OF CONTINUOUS POSITIVE AIRWAY PRESSURE AND MANDIBULAR ADVANCEMENT APPLIANCE THERAPY ON SLEEP BRUXISM IN OSA PATIENTS: A PILOT STUDY}

Deshui Li', Frank Lobbezoo', Boyuan Kuang1, Antonius A.J. Hilgevoord², Nico de Vries ${ }^{1}, 3,4$, Ghizlane Aarab1

${ }^{1}$ Department of Orofacial Pain and Dysfunction, Academic Centre for Dentistry Amsterdam (ACTA), University of Amsterdam and Vrije Universiteit Amsterdam, Amsterdam, The Netherlands; ${ }^{2}$ Department of Clinical Neurophysiology, OLVG, Amsterdam, The Netherlands; ${ }^{3}$ Department of Otorhinolaryngology, OLVG, Amsterdam, The Netherlands; ${ }^{4}$ Faculty of Medicine and Health Sciences, Department of Otorhinolaryngology, Head and Neck Surgery, Antwerp University Hospital (UZA), Edegem, Belgium.

Introduction: Sleep bruxism (SB) is characterized by rhythmic masticatory muscle activity (RMMA) during sleep. Previous studies suggested that RMMA in OSA patients is related to respiratory arousals rather than to respiratory events per se. This study aims to investigate the effect of continuous positive airway pressure (CPAP) and mandibular advancement appliance (MAA) therapy on SB in OSA patients. We hypothesized that both CPAP and MAA therapies significantly reduce RMMA in OSA patients with comorbid SB. Further, both therapies significantly reduce RMMA time-related to respiratory arousals (RMMA-RAr), while having no effect on RMMA time-related to non-respiratory arousals (RMMA-NRAr).
Methods: This cohort study included 16 OSA patients (13 males, 3 females; age $=51.5 \pm 9.1$ years; $\mathrm{AHI}=25.3 \pm 11.9$ events/hour; RMMA index $=2.9 \pm 2.3$ events/hour) who received treatment with CPAP $(\mathrm{N}=9)$ or MAA $(\mathrm{N}=7)$. Two polysomnographic recordings, one without therapy and another one with therapy (CPAP or MAA), were performed in each patient. OSA patients with an RMMA index $\geq 2$ events/hour were considered OSA patients with comorbid SB. The total RMMA indices, RMMA-RAr indices, and RMMA-NRAr indices were the primary outcome variables. Comparisons between baseline and therapy evaluation outcomes variables were made by using paired sample t-test or Wilcoxon signed-rank test in the total group of OSA patients as well as in OSA patients with comorbid SB.

Results: Both CPAP and MAA showed a significant decrease in RMMA-RAr index, the AHI, and the respiratory arousal index $(\mathrm{Z}=-3.258, P=0.001 ; \mathrm{Z}=$ $3.258, P=0.001$; and $\mathrm{Z}=-2.844, P=0.004$, respectively) in the total group. No significant changes in the total RMMA index $(\mathrm{Z}=1.008, P=0.313)$, in the RMMA-NRAr index $(\mathrm{Z}=-0.227, P=0.820)$, and in the non-respiratory arousal index $(\mathrm{T}=-0.253, P=0.804)$ were found with CPAP or MAA in situ in the total group. Nine of 16 OSA patients had comorbid SB. In these patients, both therapies significantly reduced the RMMA index $(\mathrm{T}=2.447, P=$ $0.040)$ and RMMA-RAr index $(Z=-2.666, P=0.008)$, while having no effect on RMMA-NRAr index $(Z=-$ $0.770, P=0.441)$. 
Conclusions: Both CPAP and MAA therapies significantly reduce SB in OSA patients with comorbid SB. Further, this study confirms that in OSA patients, SB is a motor response to arousals rather than to respiratory events.

Support: Not applicable.

COI Disclosures: Dr. Aarab reports grants from SomnoMed, Sunstar Suisse and VIVISOL (ResMed). The other authors have no financial conflicts of interest to declare.

\section{ABSTRACT \#002}

\section{OAT DEVICE SELECTION: CHARACTERIZING CUSTOM OAT DEVICE DESIGNS AND MATERIALS}

Jerry $\mathrm{Hu}$ DDS, D-ABDSM, D-ASBA, D-ACSDD ${ }^{1}$, Len Liptak

$\mathrm{MBA}^{2}$

\section{${ }^{1}$ Smiles of Alaska; ${ }^{2}$ ProSomnus Sleep Technologies}

Introduction: Clinical guidelines entrust OAT providers to select the custom OAT device that best minimizes side effects and optimizes treatment benefits for the patient. Though the quality of evidence supporting these guidelines is rated as low, the implication is that differences in OAT device designs and materials effect treatment outcomes.

Scant systemically collected data on OAT device design is available to providers. Providers must often rely on anecdotal information or perception when determining the suitability of a device. This investigation characterizes several OAT devices, thereby offering OAT Providers a more controlled understanding of differences in design and material performance.

Methods: One set of digital records, inclusive of upper and lower impressions and a therapeutic bite registration, were sent to four different OAT device manufacturers for the fabrication of five different types of OAT devices: Precision "P", Fulcrum Strap "FS", Traditional Dorsal "TD", Reverse Dorsal "RD" and Nylons Strap "NS".

Seven different measurements were taken for each OAT device type. Each of the seven measurements is thought to be an important consideration for OAT device selection. For example, Facial Thickness Profile is thought to be associated with lip competency.

\section{Results:}

Cross Arch Flex (mm): $\mathrm{P}=11.7, \mathrm{NS}=9.3, \mathrm{FS}=0.6, \mathrm{TD}=1.6$, $\mathrm{RD}=1.7$

Device Volume (mm displaced): $\mathrm{P}=1.1, \mathrm{NS}-2.2, \mathrm{FS}=2.8$, $\mathrm{TD}=3.8$,
Facial Profile, Maxillary (mm): $\mathrm{P}=2.2, \mathrm{FS}=6.6, \mathrm{TD}=4.2$, $\mathrm{RD}=3.4$

Facial Profile, Mandibular (mm): $\mathrm{P}=2.4, \mathrm{FS}=3.8, \mathrm{TD}=2.8$, $\mathrm{RD}=3.1$

Lingual Profile, Maxillary (mm): $\mathrm{P}=1.3, \mathrm{FS}=7.1, \mathrm{TD}=7.0$, $\mathrm{RD}=4.5$

Lingual Profile, Mandibular (mm): $\mathrm{P}=1.5, \quad \mathrm{FS}=7.6$, $\mathrm{TD}=7.4$, $\mathrm{RD}=5.1$

Titration Mechanism Profile (mm): $\mathrm{P}=7.4, \mathrm{NS}=10.1$, $\mathrm{FS}=8.5, \mathrm{TD}=9.7, \mathrm{RD}=9.1$

Conclusions: Measurable differences exist in OAT device design and material performance parameters.

- Cross Arch Flex: Precision type devices, followed by Nylon Strap type devices, exhibited more cross arch flex.

- Device Volume: Precision type devices had the smallest overall volume, followed by Nylons Strap devices.

- Facial Profile: Fulcrum Strap devices had the thickest facial profile on both the maxillary and mandibular arches. Precision OAT devices had the lowest facial profile on both upper and lower arches.

- Lingual Profile: Fulcrum Strap type devices had the thickest lingual profile on both arches. Precision type devices had the smallest lingual profile on both arches.

- Titration Mechanism Profile: The titration mechanism on the Nylon Strap type devices had the thickest profile. The titration mechanism on the Precision type device had the lowest profile.

An understanding of these differences may help OAT providers to make even better decisions matching the OAT device type with the unique needs of the patient.

Support: Smiles of Alaska was reimbursed by ProSomnus Sleep Technologies for the devices featured in this investigation.

COI Disclosures: Len Liptak is an employee of ProSomnus Sleep Technologies. 
ABSTRACT \#003

IDENTIFICATION OF DEMOGRAPHIC, ANTHROPOMETRIC, AND ACOUSTIC FACTORS ASSOCIATED WITH OBSTRUCTION SITES IN OBSTRUCTIVE SLEEP APNEA PATIENTS: A PILOT STUDY

Huang $Z^{1}$, Aarab $G^{1}$, Bosschieter $P^{2}$, Hilgevoord $A^{3}$, de Vries $N^{1,2,4}, \quad$ Lobbezoo $\quad F^{1}$

${ }^{1}$ Department of Orofacial Pain and Dysfunction, Academic Centre for Dentistry Amsterdam (ACTA), University of Amsterdam and Vrije Universiteit Amsterdam, Amsterdam, The Netherlands; '2Department of Otorhinolaryngology, OLVG West, Amsterdam, the Netherlands; ${ }^{3}$ Department of Clinical Neurophysiology, OLVG West, Amsterdam, the Netherlands; ${ }^{4}$ Faculty of Medicine and Health Sciences, Dept. of Otorhinolaryngology, Head and Neck Surgery, Antwerp University Hospital (UZA)

Introduction: Previous studies have shown that obstructive sleep apnea (OSA) patients with different obstruction sites respond differently to the same treatment. For example, tongue base obstruction was reported to be associated with positive response to oral appliance treatment (OAT), while palatal obstruction was reported to be associated with deterioration and failure to OAT. It has been hypothesized that snoring sound can be used to predict the obstruction site, facilitating the determination of treatment strategies for OSA patients. However, no study has been performed to determine the predictors of obstruction sites in OSA patients from among demographic parameters, anthropometric parameters, and acoustic characteristics of snoring sound. We hypothesized that some of these parameters can be used to predict the obstruction in the upper airway. Therefore, the aim of this study was to identify the predictors of obstruction sites in OSA patients from among demographic parameters, anthropometric parameters, and acoustic characteristics of snoring sound.

Methods: This pilot study prospectively included 62 adult OSA patients (47.4 \pm 12.2 years), with a mean apneahypopnea index (AHI) of 27. Drug-induced sleep endoscopy (DISE) and VOTE classification were used to evaluate participants' obstruction at each of the velum (V), oropharynx (O), tongue base (T), and epiglottis (E) levels. During DISE, a calibrated microphone was used to record snoring sounds and acoustic characteristics, including crest factor, dominant frequency (DF), fundamental frequency $(\mathrm{FF})$, formants (F1, F2, F3), snoring duration, and sound pressure level (max, mean, min) were extracted from each of the snoring episodes. In addition, patients' age, gender, height, body mass index (BMI), and AHI were extracted from the patients' polysomnography reports. Multilevel binary logistic regression was therefore used to identify associated factors of obstruction at each of the V, O, T, and E levels.
Results: Because 259 of 261 snoring episodes corresponded to the presence of obstruction at $\mathrm{V}$ level, the events per variable (EPV) was less than 10 and thus the logistic regression could not be performed for obstruction at $\mathrm{V}$ level. BMI $(P<0.01)$, mean $\mathrm{dB}(P=0.05)$, and FF $(P<0.01)$ showed significant associations with $\mathrm{O}$ obstruction in the univariate model, while only BMI $(P=$ $0.04 ; \mathrm{OR}=1.40[1.02-1.93])$ and $\mathrm{FF}(P=0.03 ; \mathrm{OR}=0.98$ [0.96 - 1.00]) remained significant in the multilevel model. Gender $(P=0.06)$ was the only factor that showed significant association with $\mathrm{T}$ obstruction in the univariate model, but the association became insignificant in the multilevel model. Height $(P=0.03)$ and BMI $(P=0.01)$ showed significant associations with $\mathrm{E}$ obstruction in the univariate model, but none of them remained significant in the multilevel model.

Conclusions: Based on the reported preliminary findings, we concluded that BMI is a risk factor of having obstruction at $\mathrm{O}$ level, while $\mathrm{FF}$ is a protective factor. However, no associated factor was identified for obstructions at $\mathrm{T}$ and $\mathrm{E}$ levels. Due to the small number of events, analysis could not be performed for $\mathrm{V}$ obstruction.

Support: Not applicable.

COI Disclosures: None.

\section{ABSTRACT \#004}

\section{DOSE MANAGEMENT IN DSM; ANALYSIS OF EFFICACY, STARTING POSITION, ADVANCEMENT AND SIDE EFFECTS IN A CONSECUTIVE PATIENT SERIES TREATED WITH PRECISION PLATFORM}

Mark Murphy DDS D ABDSM, Kimberly Munro Facility Director $^{2}$

\section{${ }^{1}$ Funktional Sleep; ${ }^{2}$ Funktional Sleep}

Introduction: Increased dose has long been associated with an increased risk of side effects. The utilization of a CAD CAM precision oral appliance platform that is smaller, has more tongue space, is very comfortable to wear and exhibits precision, should allow for a more conservative starting position and fewer millimeters of advancement (dose) to achieve therapeutic results. Similarly, this minimal dose therapeutic regimen would also result in fewer side effects that could lead to discontinuation of use.

Methods: 50 consecutive OSA patients (19 mild, 19 moderate and 12 severe) were treated with ProSomnus platform devices (IA, PH or CA LP) over two years. Pre and post AHI/ODI values, starting position as a percentage of the protrusive ROM, total advancement and common side effects were recorded. Starting positions were determined using $50 \%$ of the protrusive-retrusive ROM 
$+/ 1-2 \mathrm{~mm}$ depending on comfort and a negative snore sound test.

\section{Results: Efficacy}

Overall;

$76 \%$ reduction in $\mathrm{AHI} / \mathrm{ODI}$ scores from pre to post treatment.

Mild;

$71 \%$ reduction in $\mathrm{AHI} / \mathrm{ODI}, 74 \%$ under $5.0,100 \%$ under 10.0 and $95 \%$ achieved a $50 \%$ reduction from baseline.

Moderate;

$75 \%$ reduction in AHI/ODI, 58\% under 5.0, 84\% under 10.0 and $84 \%$ achieved a $50 \%$ reduction from baseline.

Severe;

$79 \%$ reduction in AHI/ODI, 27\% under 5.0, 55\% under 10.0 and $100 \%$ achieved a $50 \%$ reduction from baseline.

\section{Results: Starting Position}

The average initial starting position was $50.3 \%$ of the max protrusive max retrusive patient range. Select few were greater than $50 \%$ if a negative snore test was not achieved and they still felt comfortable advancing $1-2 \mathrm{~mm}$. A select few were less than $50 \%$ if they were not comfortable at that position.

\section{Results: Therapeutic Advancement}

The total group of 50 patents averaged $1.29 \mathrm{~mm}$ advancement from the starting position, with a range of $0.0-5.0$ $\mathrm{mm} .80 \%$ of the patients $(40)$ were in their therapeutic position within $2 \mathrm{~mm}$ of the staring position. $36 \%$ (18) resolved at the starting position. (4@+3mm, 5@+4mm and $1 @+5 \mathrm{~mm})$

\section{Results: Side Effects}

None of the patients reported discontinuation of therapy with their OA from side effects. Select patients were referred for PAP evaluation from the severe subgroup due to high residual AHI/ODI scores. $6 \%$ of patients reported transient TMD/Muscle soreness, $2 \%$ had a temporary occlusal/bite change, $6 \%$ experienced breakage to their device and $4 \%$ had a restoration removed inside the appliance that required minimal intervention. No soft tissue irritation or tooth movement was observed or reported.
Conclusions: Choosing the appropriate starting position with regards, patient comfort, protrusive range, advancement to therapeutic position and device selection are critical and effect side effects and ultimately outcomes. Smaller precision CAD CAM device platforms like the ProSomnus [IA], [IA] Select, [CA] LP, and [PH] with more tongue space can initiate therapy at more conservative positions than the literature suggest. They are also likely to experience fewer side effects that could result in discontinuation of treatment.

\section{Support: none}

COI Disclosures: Dr. Murphy is an employee and shareholder of ProSomnus Sleep Technologies.

\section{ABSTRACT \#005}

\section{EVALUATION OF DRUG-INDUCED SLEEP ENDOSCOPY AS A PATIENT SELECTION TOOL OF MAXILLOMANDIBULAR ADVANCEMENT FOR OSA PATIENTS}

Zhou $\mathrm{N}^{1,2}$, Ho JPTF', de Vries $\mathrm{N}^{2,3,4}$, de Lange $\mathrm{J}^{1}$

${ }^{1}$ Department of Oral and Maxillofacial Surgery, Academic Centre for Dentistry Amsterdam (ACTA) and Amsterdam Medical Center (AMC), Amsterdam, The Netherlands; ${ }^{2}$ Department of Orofacial Pain and Dysfunction, ACTA, University of Amsterdam and Vrije Universiteit Amsterdam; ${ }^{3}$ Department of Otorhinolaryngology, OLVG West, Amsterdam, the Netherlands; ${ }^{4}$ Faculty of Medicine and Health Sciences, Dept. of Otorhinolaryngology, Head and Neck Surgery, Antwerp University Hospital (UZA).

Introduction: Although maxillomandibular advancement (MMA) has been confirmed to be a highly effective surgical procedure for treatment of obstructive sleep apnea (OSA), further improving patient selection for MMA is called for. Drug-induced sleep endoscopy (DISE) has been confirmed to be a valid method for determining the exact site of upper airway collapse in patients with OSA. We hypothesized that DISE findings could predict the surgical outcome of MMA in OSA patients. The aim of this study was to investigate if DISE was predictive of surgical response for patients undergoing MMA for OSA.

Methods: A retrospective study was conducted on 64 patients with OSA (49 males; mean \pm SD age $=51.9 \pm 9.2$ years; mean $\pm \mathrm{SD}$ apnea-hypopnea index $[\mathrm{AHI}]=48.7 \pm$ 21.0 events/hour) who underwent baseline polysomnography (PSG) and DISE followed by MMA and subsequently 3-6-month follow-up PSG to assess treatment outcome. DISE was rated using the VOTE (velum, oropharynx, tongue, epiglottis) classification system. Responders were defined as having their AHI $<20$ events/hour with a reduction of AHI > 50\% after MMA; all others were regarded as non-responders. Preoperative and 
postoperative cephalometric analysis was performed to assess the degree of maxillary and mandibular advancement. The independent-samples t-test or Chisquare test was used to compare baseline and surgical characteristics between responders and non-responders to MMA surgery. Collapse patterns in DISE findings were compared between responders and non-responders with Chi-square test or Fisher exact test. Multivariate logistic regression was used to identified the variables that were independently associated with the response to MMA. The independent variables included in the model were those that showed significant difference in the univariate comparison between responders and non-responders.

Results: There was no significant difference at baseline between responders $(n=39)$ and non-responders $(n=25)$ regarding age, gender, body mass index (BMI), neck circumference, AHI, SNA and SNB, while the VOTE scores of responders to MMA $(5.7 \pm 1.5)$ were significantly higher than that of non-responders $(6.1 \pm 1.2)(P=0.039)$. Furthermore, no significant difference was found in degrees of maxillary and mandibular advancement between responders and non-responders. Compared to responders, non-responders had a higher occurrence of no or mild antero-posterior collapse at the level of the velum, complete circumferential collapse at the level of the velum, and complete antero-posterior collapse at the level of the epiglottis; and had a lower occurrence of complete anteroposterior collapse at the level of the velum, no or mild circumferential collapse at the level of the velum, and no or mild antero-posterior collapse at the level of the epiglottis. Multiple logistic regression analysis revealed that patients with complete antero-posterior collapse at the level of velum showed 3.46 higher odds (95\% [CI], 1.12$10.75 ; P=0.032$ ) for response to MMA, and complete antero-posterior collapse at the level of epiglottis ([OR], $0.166 ; 95 \%$ [CI], $0.040-0.684 ; P=0.013$ ) related to the nonresponse to MMA.

Conclusions: Our results suggested that DISE could be a promising tool for upfront MMA patient selection.

Support: Not applicable.

COI Disclosures: None.

\section{ABSTRACT \#006}

\section{SPLINTLESS MAXILLOMANDIBULAR ADVANCEMENT SURGERY FOR OBSTRUCTIVE SLEEP APNEA IN EDENTULOUS PATIENTS: A PILOT STUDY}

Ho JPTF1, Zhou $\mathrm{N}^{1,2}$, Klop $\mathrm{C}^{1}$, Schreurs $\mathrm{R}^{1}$, de Lange $\mathrm{J}^{1}$

1 Department of Oral and Maxillofacial Surgery, Academic Centre for Dentistry Amsterdam (ACTA) and Amsterdam Medical Center (AMC), Amsterdam, The Netherlands 2 Department of Orofacial Pain and Dysfunction, ACTA, University of Amsterdam and Vrije Universiteit Amsterdam
Introduction: Obstructive sleep apnea (OSA) is found to be prevalent in elderly edentulous subjects with an incidence of $40.9 \%$. The management of OSA in edentulous patients is challenging due to the altered anatomy and limited treatment options. Although maxillomandibular advancement (MMA) has been shown to be a highly effective surgical option for treating moderate-to-severe OSA patients, limited evidence is available regarding MMA surgery in edentulous patients. Treatment of OSA by MMA in edentulous patients is highly complicated. Therefore, the aim of this pilot study was to evaluate the clinical efficacy of MMA using a splintless treatment protocol for edentulous OSA patients.

Methods: Four consecutive edentulous patients undergoing MMA with the splintless orthognathic treatment protocol were included in this pilot study. All patients were previously unsuccessfully treated for their extreme OSA with various treatment modalities, including continuous positive air pressure (CPAP). The operations were virtually planned, followed by computer-aided design of individual osteotomy guides and patient-specific fixation implants, which were three-dimensionally printed in titanium. All patients underwent polysomnographic (PSG) recordings preoperatively and three to six months postoperatively. The surgical success was defined as apnea-hypopnea index (AHI) $<20$ events/hour with a reduction of AHI $>50 \%$ after MMA. The accuracy of this surgical technique was evaluated based on postoperative computed tomography (CT) data.

Results: First patient, 62-year-old male with baseline BMI of $33.4 \mathrm{~kg} / \mathrm{m}^{2}$, AHI of 49.8 events/hour, and lowest saturation of oxygen (LSAT) of $46.0 \%$. PSG recordings five months after MMA revealed the improvement of OSA (AHI, 16.2 events/hour; LSAT, 64.0\%).

Second patient, 61-year-old female with baseline of BMI of $31.6 \mathrm{~kg} / \mathrm{m}^{2}$, AHI of 82.9 events/hour, and SAT of $72.0 \%$. PSG recordings three months after MMA revealed the improvement of OSA (AHI, 8.3 events/hour; LSAT, $79.0 \%)$.

Third patient, 60-year-old male with baseline of BMI of $30.4 \mathrm{~kg} / \mathrm{m}^{2}$, AHI of 90.0 events/hour, and LSAT of $78.0 \%$. PSG recordings three months after MMA revealed the improvement of OSA (AHI, 17.8 events/hour; LSAT, $89 \%)$.

Fourth patient, 58-year-old female with baseline of BMI of $30.5 \mathrm{~kg} / \mathrm{m}^{2}$, AHI of 93.5 events/hour, and LSAT of $61.0 \%$. PSG recordings six months after MMA revealed the improvement of OSA (AHI, 9.7 events/hour; LSAT, $88.0 \%)$.

All four patients were successfully treated according to the success criteria. The mean achieved advancement of the 
maxilla was $9.0 \pm 2.5 \mathrm{~mm}$ (range $6.5-12.3$ ). For the maxilla there was a mean translation discrepancy of 0.6 $\mathrm{mm}$. For the rotation there was a mean discrepancy of $2.4^{\circ}$, $0.2^{\circ}, 0.4^{\circ}$ for the pitch, yaw and roll respectively. The mean discrepancy in translation of the mandible was $0.5 \mathrm{~mm}$.

Conclusions: Based on the preliminary findings, we conclude that MMA surgery with the use of the presented splintless treatment protocol is an effective and predictable treatment strategy aimed at edentulous patients with OSA.

Support: Not applicable.

COI Disclosures: None.

\section{CASE REPORT \#007}

\section{PERILS OF IMPLANT-SUPPORTED ORAL APPLIANCE} IN A FULLY EDENTULOUS PATIENT- A CASE REPORT

Jayaraman, Sripriya ${ }^{1}$, Eisa, Elfatih ${ }^{2}$ Moreno-Hay, Isabel ${ }^{2}$

\section{${ }^{1}$ Private Practice, $C A ;{ }^{2}$ University of Kentucky, $K Y$}

Introduction: Mandibular advancement device (MAD) is the preferred treatment option for primary snoring and is considered a viable alternative to treat obstructive sleep apnea (OSA) with varying severities. While this may be true, the application of MAD can be especially challenging with edentulism. Over the years, dental implant therapy has been increasingly preferred for managing missing teeth as it can improve one's function and quality of life. Loading intensification on implants due to MAD is a concerning issue. Finite element analysis has shown that maximum forces are concentrated in the second molar areas with natural dentition and dental implants. It can be inferred that increased loading might lead to implant failure.

Report of Case: A 73-old male reported to the Orofacial Pain Clinic in October 2019 for MAD therapy trial due to intolerance to Positive Airway Pressure therapy. He presented with a home sleep study, conducted in January 2017, revealing an AHI of 24 and an oxygen saturation level of $71.3 \%$, consistent with moderate OSA. His clinical examination showed a BMI of 36.7 and a neck circumference of 19 inches. His maximum mouth opening was $50 \mathrm{~mm}$, with a protrusive range of $13 \mathrm{~mm}$. Intraoral exam revealed multiple missing teeth, and his remaining teeth exhibited poor prognosis. The restorative dentist decided that the best course of action was to extract all remaining teeth and restore his dentition with a fixed fullarch implant-retained prosthesis: all-on-6 implants in the maxilla, and all-on-5 implants in the mandible. Implant placement was completed seven months before the initiation of MAD therapy. Prior to MAD therapy, the patient faced a restorative complication involving debonding of tooth \#10, that was successfully repaired. At the time MAD initiation, implant bearing and supporting areas appeared healthy. Shim stock exam revealed stable occlusal contacts. His treatment with MAD (Herbst type) was initiated. The patient's final therapeutic position was determined at $75 \%$ of maximum protrusive capacity. During the first six-months of treatment, \#10 denture tooth was debonded and repaired again. In an eight-month visit, the patient voiced his contentment with the treatment. He denied having any side-effects caused by the device. He reported 95\% improvement in his sleep quality, daytime fatigue, and sleepiness. His examination uncovered a BMI of 35.6, clinically serviceable restorations and stable occlusal contacts. Panoramic X-ray showed no bony changes in implants since the start of MAD therapy.

Discussion: $17 \%$ rate of mechanical failure (like fracture or loosening of artificial teeth) has been reported in the literature. Different framework designs have been associated with treatment success. Combined with finite element analysis prediction of force concentration, we can hypothesize that MAD with implant retained prosthesis could have varying complications. To minimize implant complications, knowledge of stress concentration areas, framework designs, and possible failure patterns could help clinicians plan for improved implant placement and modify MAD accordingly. Therefore, effective communication with the restorative dentist is essential. Clear communication with the patient and understanding the patient's expectation are equally crucial for treatment success.

\section{Support: None.}

COI Disclosures: None.

\section{CASE REPORT \#008}

\section{MULTIDISCIPLINARY INTERVENTION AND THERAPY ON A PEDIATRIC OBSTRUCTIVE SLEEP APNEA: A CASE REPORT}

Falardo Ramos, S. ${ }^{1}$ Marques, S. ${ }^{2}$

\section{${ }^{1} D D S, M S c, P h D$, Qualified Dentist EADSM, International Certificant ABDSM \\ ${ }^{2} \mathrm{MD}$, Sleep Medicine Expert ESRS, PNL-starter}

Introduction: Pediatric Obstructive Sleep Apnea can be extremely challenging due to the presence of multi-system symptomatology, namely at the behavioral, cognitive and physical level. Need a team of experts capable of recognizing symptoms associated with poor sleep and available for a multidisciplinary intervention, including educational psychology, pediatric nutritionist, family support, Oral Myofunctional Therapy and Interceptive Orthodontic.

Report of Case: The authors present a clinical case of a short 11-year-old girl with low self-esteem, dysfunctional behavior and poor school performance, suspected of leaky gut and with no evident complaints of poor 
sleep. Considering the clinical history and the Intra-Oral Exam of a class II molar and canine a severe overbite, Mallampati Class IV, hypertrophic tonsils, tongue muscle hypertonus, edentulous tongue, dental signs of bruxism, uvula ptosis, the patient undertook an analytic evaluation and a sleep study, which revealed deficiencies of iron, vitamin $\mathrm{D}$, folic acid and magnesium, as well as severe sleep apnea. In a more detailed Facial Prefile and Postural Evaluation we can easily identify an anterior head and cervical posture, a concave facial prefile, short mandible, anterior rotation of the scapula's, sternum spectrum cavum, internal rotation of the knees and feet. The Cephalometric Analysis, revealed a skeletal class II, a severe retrognatic mandible, a brachycephaly craneofacial growth. On the Oral Myofunctional Evaluation, mild labial tonus, lip sealing with contraction of the mentonian muscle, difficulty in performing lingual praxis exercises. Recommended for Treatment Plan: After excluding surgical indication by an otorhinolaryngologist, initiated Twin block and myofunctional exercises, as well as correction of vitamins and minerals, reduction of carbohydrates, improved sleep habits and support of educational psychologists with sleep experience.

Discussion: After two years of excellent adhering of a combined therapy, this girl improved growth, achieved a good integration with her classmates and overcame school difficulties and the control sleep study revealed the total correction of the sleep apnea. Keeps supplementation in progress and personalized nutrition. The authors stress the importance that an early intervention at the orthodontic and myofunctional level, reveals to be an excellent treatment option with excellent results in POSA, eliminating AHI, improving ODI, posture, breath and muscle tone. The outcomes on combined therapy has positive implications for quality of life, school performance at the cognitive and behavioral level.

Support: No applicable.

COI Disclosures: None.

\section{ABSTRACT \#009}

\section{COMPARISON OF FOUR MANDIBULAR ADVANCEMENT DEVICES IN THE MANAGEMENT OF OBSTRUCTIVE SLEEP APNEA.}

Linda Sangalli ${ }^{1}$, Fernanda Yanez Regonesi ${ }^{1}$, Diego Fernandez Vial ${ }^{1}$, Andres Martinez Porras ${ }^{1}$, Isabel Moreno Hay ${ }^{1}$

\section{Orofacial Pain Clinic, University of Kentucky, Lexington, USA}

Introduction: Mandibular advancement device (MAD) is recognized as an effective treatment in management of mild-moderate Obstructive Sleep Apnea (OSA) or in lack of tolerance to CPAP. Systematic review (Ahrens, 2011) found that all MADs improved polysomnographic indices, compared with inactive appliances, custom-made monobloc were more effective than thermoplastic monobloc. To the best of our knowledge, only few studies assessed the differences in effectiveness between designs of MADs: except for two studies (Gauthier, 2009; Rose, 2002), no significative difference was found between other types of MAD (Verburg, 2018; Bloch 2000; Lawton, 2005). The aim of the study was to investigate the effectiveness of four different designs of MAD, comparing AHI scores and minimum blood oxygen saturation (min $\mathrm{SpO}_{2}$ ) values between pre- and post-treatment studies.

Methods: Participants referred to the Orofacial Pain Clinic at University of Kentucky between July 2007 and June 2020 for the treatment of OSA with MAD were evaluated. Only retrospective data of participants with a second sleep study with MAD were included. For analysis, two different criteria for improvement in OSA were established. The first was set at 50\% improvement in AHI. The second was set at 50\% improvement in AHI, with AHI below 10/h. Treatment failure was defined as an increased AHI after MAD therapy. Wilcoxon Signed Rank test and Kruskal Wallis test were performed to compare pre- and posttreatment data and the effectiveness of the four appliances, respectively.

Results: Among 462 patients in treatment with MAD, 393 were excluded due to lack of post-treatment sleep study. Final sample comprised 69 patients (34 women and 35 men) with pre-treatment AHI of 22.26 \pm 16.41 . At baseline, 23 patients showed mild OSA (AHI $8.7 \pm 2.48$ ), 32 moderate OSA (AHI $20.36 \pm 4.29$ ), 14 severe OSA (AHI $47.21 \pm 18.97) .23$ were treated with Dorsal-A $(33.33 \%)$, $21(30.43 \%)$ with Herbst, 18 (26.10\%) with Dorsal-B, and $7(10.14 \%)$ with CAD-CAM appliances. The results showed an overall decrease in AHI, with a post-treatment AHI 10.41 \pm 18.37 . The improvement was statistically significant with Dorsal-A, Herbst, Dorsal-B and CADCAM appliances $(\mathrm{p}=.016, .008, .017$ and .043 respectively). No statistically significant difference was found between the four appliances $(\mathrm{p}=.774)$. Treatment success (first criteria) was obtained in 37 patients (53.62). Treatment success (second criteria) was achieved in 30 patients $(43.47 \%)$. No statistically significant difference was found between the four appliances in treatment success $(\mathrm{p}=.451)$. Treatment failure was noticed in 16 subjects (23\%): 4 (17.39\%) with Dorsal-A, 5 (23.80\%) with Herbst, $6(33.33 \%)$ with Dorsal-B, 1(14.28\%) with CAD-CAM appliance. No significant difference in minimum $\mathrm{SpO}_{2}$ was found between the four appliances $(\mathrm{p}=0.88)$.

Conclusions: Based on results of this study, the four different MAD designs were effective and in improving AHI. MAD should be selected on an individual basis, according to patient preference and trained dentist recommendation. Further analysis is needed to determine 
the percentage of protrusion at which effective treatment is achieved between appliances. Study also highlighted importance of post-treatment sleep study, as some patients showed a worsening in AHI score.

\section{Support: None}

COI Disclosures: None.

\section{CASE REPORT \#010}

\section{EFFECTIVENESS OF MANDIBULAR ADVANCEMENT DEVICE IN SEVERE OBSTRUCTIVE SLEEP APNEA}

Patricia Guerrero, DDS, Leopoldo P. Correa, BDS, MS.

\section{Tufts University School of Dental Medicine.}

Introduction: Obstructive sleep apnea (OSA) is a common condition affecting roughly 1 in 7 of the world's population. The management of patients with OSA requires a multidisciplinary approach with many treatment options currently available. OSA has been effectively managed with positive air pressure (PAP) since the early 1980 's, and is considered first line of treatment for severe OSA patients. Many patients find PAP therapy cumbersome with compliance decreasing over time. According to current guidelines, mandibular advancement devices (MAD's) are indicated for patients with mild to moderate OSA. This case report demonstrates successful management of severe OSA with MAD therapy when anthropomorphic factors along with sleep study data and clinical expertise of the provider are carefully considered before treatment.

Report of Case: This case report portrays a 55-year-old female diagnosed by sleep physician with severe OSA $\left(\mathrm{AHI}=37\right.$ and $\left.84 \% \mathrm{O}^{2}\right)$ while subjectively reporting 8/24 on the Epworth Sleepiness Scale (ESS). Initially, she attempted PAP therapy as first line of treatment for this level of severity. However, she was intolerant to the PAP machine and was consequently referred to Tufts Dental Sleep Clinic for assessment and therapy with MAD. Upon clinical history and examination, a BMI of 23.5 was revealed while her neck circumference measured 12.5", and cephalometric analysis depicted a low mandibular angle and reduced hyoid bone-to-mandible distance. Impressions and bite registration were completed, and a bilateral interlocking design MAD was fabricated with $80 \%$ of mandibular advancement as a starting point. Sleep device and morning repositioning aligner were then delivered. Patient returned for follow up appointments to assess changes in symptoms; no additional titration of oral device was needed and patient reported no side effects. After completion of the MAD clinical protocol, she was referred back to her sleep physician for a follow up sleep study which objectively revealed a reduction to normal values of respiratory events $\left(\mathrm{AHI}<5\right.$ and $91 \% \mathrm{O}^{2}$ ) while reporting 0/24 on the ESS. Long term follow-up was implemented as standard of dental sleep medicine clinical care.

Discussion: Clinical practice guidelines recommend that sleep physicians prescribe MAD's for adult patients who are intolerant to PAP therapy. MAD efficacy in severe OSA cases is reserved and requires an understanding of patient characteristics and predictors of success. This case report demonstrates successful management of severe OSA with MAD therapy when anthropomorphic factors along with sleep study data and clinical expertise of the provider are carefully considered before treatment.

Support: Authors declared no conflict of interest and no financial support provided for this case report.

\section{COI Disclosures: None.}

\section{ABSTRACT \#011}

\section{PRECISION ORAL APPLIANCE THERAPY: THE PRIME -TIME TREATMENT FOR OSA}

\section{Edward T Sall, MD DDS MBA}

Introduction: A growing body of research has established that Oral Appliance Therapy delivers non-inferior outcomes in comparison to CPAP. CPAP was previously considered the "gold standard" due to its high efficacy in eliminating obstructive events. However, despite improvements in technology, the "effectiveness" of CPAP has been compromised by poor real- world compliance. At the same time, technological improvements have endeavored to improve the "effectiveness" of Oral Appliance Therapy. This study reports the efficacy of a novel, precision engineered Oral Appliance Therapy device for the primary treatment of all severities of OSA.

Methods: Digital Scans (Trios 3 Shape) and digital bite registrations were recorded with a George Gauge utilizing a $3 \mathrm{~mm}$ bite fork and were obtained on all patients. Initial protrusion was set at $40-60 \%$ and was varied according to the degree of overbite and overjet, the severity of the OSA, and the presence or absence of TMJ symptoms. All patients were fitted with a ProSomnus $\{\mathrm{IA}\}$ MAD and titrated according to their subjective symptoms (snoring, hypersomnolence, jaw discomfort). An efficacy study was obtained with a HST after titration and compared to the initial HST or PSG.

Results: Outcome data on 85 patients ( 37 females and 48 Males, aged 56.7+/- 10.2 years was retrospectively reviewed. The patients were consecutively ordered with complete follow up data, many patients did not return for follow ups. The pre-treatment AHI was $24.5+/-19.1$ with 36 Mild, 26 Moderate and 23 Severe patients. O2 Nadir was $84.1 \%+/-5.5 \%$. Post-treatment AHI was $5.5+/-4.9$ with an AHI reduction of $71.6 \%+/-20.3 \%$. $59 \%$ of the 
patients scored $<5,31 \%<10$ and $10 \%>10$ on their outcome AHI data. O2 Nadir improve to $88.2 \%$ overall. 31 patients had REAR's (RDI-AHI) $>10$ with an average of $16.7+/-7.5$, of these patients 27 saw a reduction of REAR's of $54.4 \%$ from 16.9 to 7.1 .

Conclusions: The data shows that a precision oral appliance is capable of successfully treating patients with all levels of severity, with the majority of patients treated to an AHI <5. 23 severe patients with an average AHI of 49.7 were treated to a final average of $8.6,4$ of the patients scored above 10. Additionally, patients successfully saw a reduction in upper airway resistance airflow as evidenced by the reduction of RERA's of $54.5 \%$ showing that a precision oral appliance can have a significant impact on the upper airway.

Support: The data support the digital workflow and treatment of OSA with a precision OA across all levels of OSA. There was no industry support for this study.

COI Disclosures: Dr. Sall is the Medical Director of Prosomnus Sleep Technologies and Medical Director of Better Night Solutions.

\section{ABSTRACT \#012}

\section{WHAT DO WE KNOW ABOUT SLEEP MEDICINE PHYSICIANS?}

Len Liptak, MBA ${ }^{1}$

\section{ProSomnus Sleep Technologies}

Introduction: Minimal systemic information exists on sleep medicine physicians (SMPs). Given that SMPs play an essential role in the diagnosis of OSA and therapy selection, this investigation offers a more comprehensive characterization of SMPs, and their attitudes and behaviors regarding OAT.

Methods: Fletcher Spaght International was commissioned by ProSomnus Sleep Technologies to conduct a blinded, evaluation of SMPs. The respondent $\begin{array}{llll}\text { quota } & \text { was } & 150 & \end{array}$

Inclusion criteria was as follows:

- Must have diagnosed at least 50 people with OSA in the prior 12 months;

- At least $50 \%$ of the sample must be Board Certified in Sleep Medicine;

- No more than $15 \%$ of the sample spends less than $25 \%$ on sleep medicine patient care.

\section{Results:}

SMP Characteristics

- $39 \%$ pulmonology, $32 \%$ internal medicine or primary care, $15 \%$ neurology, $14 \%$ ENT

- $53 \%$ were not board certified, $47 \%$ were board certified

- $75 \%$ were in private practice, $25 \%$ were in academic institutions

- Sleep medicine physicians diagnosed 327 OSA patients per year, on average

- $50 \%$ of respondents spend less than $50 \%$ of time on sleep medicine; $11 \%$ report spending $100 \%$ of their time on sleep medicine

- $33 \%$ of patients are severe, $28 \%$ moderate, $29 \%$ mild

- $81 \%$ expressed interest in learning more about new OAT technologies and protocols

SMP Perspectives on Therapies

- CPAP accounted for $75 \%$ of prescriptions, $25 \%$ was spread amongst OAT, Upper Airway Neurostimulation and others

- $68 \%$ reported that their patients were satisfied with CPAP; $50 \%$ for OAT; $54 \%$ for Neurostim

- $63 \%$ reported that their patients successfully adopt CPAP, 54\% successfully adopt OAT

- $32 \%$ of SMPs expected to increase OAT prescriptions over the next 3-5 years, $9 \%$ expect to decrease OAT prescriptions

\section{SMP Barriers to Increasing OAT Prescriptions}

- $94 \%$ cited lack of insurance coverage and reimbursement (private and Medicare)

- $88 \%$ cited unpredictable, variable, performance of the OAT device

- $88 \%$ cited side effects of OAT

- $86 \%$ cited lack of compliance monitoring and data

- $83 \%$ cited availability of qualified sleep dentists

- $82 \%$ cited lengthy time to treatment with OAT

- $81 \%$ cited unpredictable everyday symptom alleviation

- $78 \%$ cited patient adherence (OAT painful, difficult to use, not reliable, break frequently)

Conclusions: SMPs have an evolving and varied understanding of OAT. SMPs reported that fewer patients are satisfied and successfully adopt OAT relative to CPAP. However many SMPs also reported that they expect to treat 
more patients with OAT in the coming years.

SMPs also reported a willingness to treat a greater number of patients with OAT if key barriers are addressed. The clinically oriented barriers to prescribing more OAT (eg insurance reimbursement, access to qualified dentists, etc) may be addressed through better advocacy and education. The OAT device specific barriers (compliance monitoring, unpredictable performance, side effects, etc.) to more prescriptions may be addressed through better OAT device selection and advances in therapeutic, diagnostic and theragnostic technologies.

Support: This investigation was sponsored by ProSomnus Sleep Technologies.

COI Disclosures: Len Liptak is an employee of Prosomnus Sleep Technologies.

\section{ABSTRACT \#013}

\section{IMPROVEMENT IN NOCTURIA IN OBSTRUCTIVE SLEEP APNEA PATIENTS WITH THE USE OF MANDIBULAR ADVANCEMENT DEVICE.}

Fernanda Yanez-Regonesi, Linda Sangalli, Andres Martinez-Porras, Diego Fernandez-Vial, Isabel MorenoHay

Orofacial Pain Clinic, University of Kentucky, Lexington, USA

Introduction: Nocturia is defined by the the International Continence Society as "waking up at least two times for voiding". There are reports in the literature of the relationship between obstructive sleep apnea (OSA) and nocturia. In fact, the prevalence of nocturia among OSA patients varies between $45-70 \%$ depending on the severity of OSA and is considered as a predictor or indicator of risk for OSA. When comparing sex differences, Saldias et al. (2019) reported to be slightly more prevalent in females (males 60.4\%, females 64.8\%), becoming a more significant difference in the study of Basoglu et al., (2018) where the prevalence was $54 \%$ in females versus $41 \%$ in males. Miyazaki et al. (2015) concluded that CPAP treatment improved nocturnal voiding, probably owing to normalization of oxygen desaturation caused by a significantly reduced AHI. A systematic review and metaanalysis by Wang et al. (2015) included 307 patients and compared the nocturia before and after CPAP treatment. The group concluded that patients with OSA and nocturia who were treated with CPAP had a significant decrease in the frequency of nocturia and the volume of urine associated with it. To the best of our knowledge, only one study has investigated the correlation between nocturia and mandibular advancement device (MAD) treatment. Attali et al. (2016) reported a significant short-term reduction in nocturia, from a prevalence of $48 \%$ at the beginning of the study to $13 \%$ after 3-6 months treatment. The treatment was less efficient in long-term control, likely as a result of worsening of OSA severity. The aim of this study was to investigate the effect of MAD in the prevalence of nocturia.

Methods: Participants referred to the Orofacial Pain Clinic at the University of Kentucky between September of 2017 and July of 2020 for the treatment of OSA with MAD were evaluated for nocturia. During their first appointment they were asked how many times they wake up at night to urinate, and the same question was repeated during follow ups. Additionally, medical records were obtained to evaluate for any prostate/kidney problems. Paired T-test was performed to evaluate any significant difference between baseline and third follow up visit.

Results: 98 records were evaluated. Of those, 4 were eliminated due to incomplete data. 30 out of the 94 $(31.91 \%)$ presented nocturia at baseline. Of those $60 \%$ (18/30) were females and 40\% (12/30) males. $3 / 30$ presented prostate/kidney problems. The average followup appointment was $6 \pm 3.65$ months. $46.6 \%$ of the participants were given a Dorsal appliance, $30 \%$ a Panthera appliance and $22.3 \%$ a Herbst appliance. Significant improvement in nocturia was noted with the use of MAD, $t(29)=2.152, P=.04$. This remained significant after excluding the participants with prostate/kidney problems, $\mathrm{t}(26)=2.167, \mathrm{P}=.04$. $17 / 30(56.6 \%)$ no longer presented nocturia at the third follow up.

Conclusions: Based on the results of this study, MAD therapy provides significant improvement in nocturia secondary to OSA. Further studies are needed to determine if the improvement in nocturia is correlated with the level of protrusion and with the improvement in AHI.

Support: None.

COI Disclosures: None.

ABSTRACT \#014

REMOTELY CONTROLLED MANDIBULAR
POSITIONING OF ORAL APPLIANCE THERAPY
DURING DRUG-INDUCED SLEEP ENDOSCOPY AND
POLYSOMNOGRAPHY COMPARED
CONVENTIONAL WITH
PATIENTS WITH OBSTRUCTIVE SLEEP APNEA

Elahe Kazemeini, MD 1,2;Sara Op de Beeck, MSc, PhD1,2; Anneclaire Vroegop, MD, PhD ${ }^{1,2}$; Dorine Van Loo, MSc ${ }^{2}$; Marc Willemen, MSc ${ }^{5}$; Johan Verbraecken, MD, $\mathrm{PhD}^{4,5}$; Marc J Braem, DDS, PhD ${ }^{1,3}$;Olivier M. Vanderveken, MD, $\mathrm{PhD}^{1,2,4}$;Marijke Dieltjens, MSc, $\mathrm{PhD}^{1,2}$,

${ }^{1}$ Translational Neurosciences, Faculty of Medicine and Health Sciences, University of Antwerp, Wilrijk, Antwerp, Belgium; '2Department of Otorhinolaryngology, Head and Neck Surgery, Antwerp University Hospital, Edegem, 
Antwerp, Belgium; ${ }^{3}$ Department of Special Dentistry Care, Antwerp University Hospital, Edegem, Antwerp, Belgium; ${ }^{4}$ Multidisciplinary Sleep Disorders Centre, Antwerp University Hospital, Edegem, Belgium; ${ }^{5}$ Department of Pulmonary Medicine, Antwerp University Hospital, Edegem, Belgium

Introduction: One of the most commonly used non-CPAP therapies for obstructive sleep apnea (OSA) is a titratable custom-made mandibular advancement device (MAD). MAD has a lower efficacy to CPAP while the higher adherence to MAD counterbalances its lower efficacy leading to equal overall clinical effectiveness. Therefore, improving the efficacy of MAD-therapy is of utmost importance. A major determinant is the amount of mandibular protrusion, yet the optimal titration protocol remains unclear. Currently, the effective target protrusiveposition (ETPP) is achieved by "subjective-titration", based on gradual advancement of the mandible guided by subjective improvement of symptoms. However, this process relies on patient cooperation and can take a longer time. This study compared subjective-titration, polysomnography (PSG)-guided titration and druginduced sleep endoscopy (DISE)-assisted titration of MAD for the determination of the ETPP. For both PSG-guided and DISE-assisted-titrations, a remotely controlled mandibular positioner (RCMP) device was used.

Methods: In this ongoing prospective randomized crossover clinical trial, patients undergo three different titrations: subjective-titration, PSG-guided-titration and DISE-assisted-titration, both using an RCMP device. After each method, the patient was treated with the MAD in the corresponding ETPP as determined by that respective titration method, for one month, followed by a PSG with MAD. Outcomes were the protrusion from the edge-toedge incisor position $(\mathrm{mm})$ and the apnea/hypopnea-index (AHI) with MAD in the selected ETPP as compared to baseline AHI. Data are presented as median and interquartile-ranges. Pairwise comparisons between methods were performed. After Bonferroni correction, statistical significance was considered if $\mathrm{p}<0.017$.

Results: Currently, ten patients $(\mathrm{n}=10$; age:47.7 years; $90 \%$ male; $\quad \mathrm{BMI}=30.2 \mathrm{~kg} / \mathrm{m}^{2}[27.2-31.2]$; baseline $\mathrm{AHI}=21.4 / \mathrm{h}[17.5-26.8]$ ) are included in this study. Subjective-titration was performed over a one-month time period, while PSG-guided-titration was performed overnight and DISE-assisted-titration within one DISE session. The ETPP was collected in all 10 patients for the 3 titration methods $(\mathrm{N}=30)$. The median ETPP was 12.1mm[11.1-13.6], 11.1mm[10.6-12.1] and 11.1mm[7.813.4] for subjective-titration, PSG-guided-titration and DISE-assisted-titration, respectively. Pairwise comparisons revealed no statistical difference in ETPP among these methods (subjective vs PSG $\mathrm{p}=0.1444$; subjective vs DISE $\mathrm{P}=1$; PSG vs DISE $\mathrm{P}=$ $0.1413)$.
Up till now, five subjects had follow-up PSG in all 3 different ETPPs corresponding to the respective titration method. The baseline AHI decreased from 25.3/h[24-27.3] to $6.5 / \mathrm{h}$ [5.6-11.2] with MAD after subjective-titration; to $5 / \mathrm{h}[2.4-12.7]$ after PSG-guided-titration and to $7.7 / \mathrm{h}[6.1$ 11.1] after DISE-assisted-titration. AHI reduction was not significantly different among the three protocols (subjective vs $P S G \mathrm{P}=0.1837$; subjective $\quad v s$ DISE $\mathrm{P}=0.7409$; PSG vs DISE $\mathrm{P}=0.553$ ).

Conclusions: In this clinical trial, there was no significant difference in mandibular ETPP or AHI reduction among the three different titration methods. These findings validate the efficacy of DISE-titration in determining the ETPP and in upfront patient selection for MAD therapy. Furthermore, DISE-titration is time-efficient in comparison to both other approaches, considering the timeconsuming 'trial and error' procedure of the subjectivetitration or an over-night-PSG. Additionally, subjectivetitration has the disadvantage, compared to PSG-guidedtitration and the DISE-assisted-titration, of a high cost of starting the therapy in predicted non-responders. In conclusion, our results suggest that the objective methods are of surplus value to optimize MAD treatment in OSA patients.

Support: This study was supported by the research foundation Flanders, as part of the postdoctoral project of Prof. dr. Marijke Dieltjens (project number: 12H4520N).

COI Disclosures: Dr. Braem sits on the advisory board of Somnomed and Resmed and holds the research chair of Somnomed. Dr. Vanderveken is a Member of the Medical Advisory Board of Zephyr Sleep Technologies - Promotor of Somnomed Grant at Antwerp University Hospital Promotor of Research Grant from Philips at Antwerp University Hospital and reports research support at Antwerp University Hospital from Inspire Medical Systems and Nyxoah.

\section{ABSTRACT \#015}

\section{THE COSTS OF OBSTRUCTIVE SLEEP APNEA TREATMENTS: IS OAT MORE EXPENSIVE?}

Len Liptak, MBA ${ }^{1}$

\section{ProSomnus Sleep Technologies}

Introduction: The keynote lecture for the 2019 World Sleep Congress concluded that Oral Appliance Therapy (OAT) for the treatment of Obstructive Sleep Apnea (OSA) was ready for prime time and that OAT providers make too much. The purpose of this investigation is to quantify the economic burden rate associated with two popular OSA treatment modalities: CPAP and OAT.

Methods: Fee schedules were procured from the Centers for Medicare Services for CPAP and Oral Appliance 
Therapy treatment modalities. Simple averages were used to account for regional, and other, differences in reimbursement rates. Replacement interval durations were identified. Publicly available device warranty durations were used when the fee schedule did not specify a replacement

duration.

The daily reimbursement burden rate was calculated by dividing the reimbursement fee for each item by the replacement interval duration.

Results: The daily reimbursement burden rate for a standard CPAP system was \$2.99. The system includes: Mask@ \$1.00/day, Mask Cushion@ @ 1.15/day, Mask Nasal Pillows @ \$0.51/day, Tubing @ \$0.13/day, Headgear@ @0.10/day, Disposable Filters @ \$0.07/day, CPAP Machine (standard) @ \$0.04/day.

The daily reimbursement burden rate for OAT was $\$ 0.77$.

Conclusions: In this analysis, CPAP was 3.9 times more expensive per day than OAT.

It is important to consider that the reimbursement architectures for CPAP and OAT are very different. The CPAP reimbursement structure is comprised of a series of codes, each with different reimbursement amounts and replacement schedules. The OAT reimbursement structure is singular, and it is inclusive of the device and the professional services associated with administering, adjusting and monitoring treatment over a five-year duration.

There are limitations to this study. The reimbursement burden for CPAP could vary significantly based on the needs of the patient. For example, the cost per day increases to $\$ 3.20 /$ day, 4.2 times more expensive than $\mathrm{OAT}$, if the patient requires a Bilevel CPAP machine.

The relationship between replacement schedules and device warranty duration should also be considered in future studies. For example, at the time of this investigation only one Medicare verified OAT device, a precision Herbst-style device, had a warranty period that matched the reimbursement schedule. All other Medicare verified OAT devices had warranty durations of three years or less. This raises the question of who absorbs the cost burden if the device fails after the warranty has expired but before a new device

covered?

Lastly, the daily reimbursement burden rates for both CPAP and OAT treatment modalities may vary based on private insurance reimbursements rates, regional differences, and other factors.

Support: This investigation was sponsored by ProSomnus Sleep Technologies.
COI Disclosures: Len Liptak is an employee of Prosomnus Sleep Technologies.

\section{CASE REPORT \#016}

\section{CASE STUDY ON TOUGHNESS OF MULTIPLE DEVICES WITH A SEVERE BRUXING PATIENT}

Neal Seltzer DMD ${ }^{1}$, Gina Pepitone-Mattiello $\mathrm{RDH}^{2}$, Keith Batcheller $^{3}$

${ }^{1}$ Long Island Dental Sleep Medicine: Oral Appliance Therapy for Snoring and Sleep Apnea; ${ }^{2}$ Long Island Dental Sleep Medicine: Oral Appliance Therapy for Snoring and Sleep Apnea; ${ }^{3}$ Vice President Product Marketing: ProSomnus Sleep Technologies

Introduction: Oral appliance therapy (OAT) for obstructive sleep apnea (OSA) has gained prevalence in recent years. The problems facing dentists are those patients which exhibit a combination of bruxing in the midst of being treated for OSA which result in the breakage of many of the current OSA appliances on the market today. This case study will provide insight into viable OAT devices that have withstood excessive forces, resulted in successful oral appliance therapy and satisfied patients.

Report of Case: In 2015 patient "Z" a 28-year-old male with a BMI of 25.6 and weight of 205 presented increased snoring, had PSG and was diagnosed with OSA but the patient was not confident in the results or in the physician conducting the study. He then in 2016 was treated by an Otolaryngologist and elected surgery for a deviated septum. Post-surgical observations revealed ongoing snoring with EDS and was still fatigued after 9 hours of sleep

In November 2017, patient " $Z$ " came to our office and was previously diagnosed with Asperger syndrome and sleep bruxism. Records and impressions were taken for an oral appliance. His starting sleep vitals were the following: AHI:17.1, Supine:35.2 and NADIR: 82.

Over the course of almost 3 years the patient was provided different types of oral appliances including a $1^{\text {st }}$ and $2^{\text {nd }}$ generation continuous advancement device, a lowprofile precision iterative advancement medical device and a herbst-style medical device.

In all cases the patient caused torqueing of the sliding posts, loosening and weakening of the continuous advancement device resulting in breakage and or bending and breakage of the herbst style arm and screws.

In the Summer of 2020, the patient was provided a milled oral device with a soft liner and fulcrum strap in hopes this sleep device would prove successful with little to no breakage. The device was successful in no breakage but 
due to the patient's wide range in mouth opening the device continued to pop off the lower arch.

Then in October of 2020 we delivered a new Novel Iterative Advancement Device (NIAD) from ProSomnus ${ }^{\text {TM }}$ which was made of a new medical grade six material which was $8 \mathrm{x}$ more durable than the previous control cured, milled PMMA material. It exhibited high tensile strength, comfort, and provided our office a robust iterative alternative to all other devices.

The patient now has reported that he sleeps well with no breakage and prefers this device to all others. He reports a reduction in snoring by $50-60 \%$. His last reported sleep vitals were: AHI 3.4, Supine: 5.6 and NADIR: 90.

In total patient " $Z$ " went through 17 product performance or breakage issues until the milled oral, soft liner device and the new medical grade six, precision, high tensile strength OA became available during an IRB study.

Discussion: The use of precision, easy delivery, and robust high tensile strength materials for OSA patients presenting severe bruxism will be critical in successful treatments of these unique individuals. The proper diagnosis of bruxism early in the process will save much time, cost and reduce patient frustration with the delivery of these new robust oral appliances.

Support: Devices provided at no charge by ProSomnus and SomnoMed.

COI Disclosures: Keith Batcheller is an employee of ProSomnus Sleep Technologies.

\section{CASE REPORT \#017}

\section{MANAGEMENT OF HYPOGLOSSAL NERVE STIMULA- TION COMPLICATION: A MULTIDISCIPLINARY AP- PROACH}

Aaron Glick, DDS ${ }^{1}$, Jerald Simmons, $\mathrm{MD}^{2,3}$

1 University of Texas Health Science Center at Houston School of Dentistry, Houston, TX ${ }^{2}$ Comprehensive Sleep Medicine Associates, Houston, TX ${ }^{3}$ Sleep Education Consortium

Introduction: Hypoglossal nerve stimulation (HGNS) is a relatively new treatment modality for obstructive sleep apnea (OSA) that was FDA approved in 2014. Patients receiving HGNS undergo a surgery to implant a respiration sensor in the chest wall, a small impulse generator beneath the clavicle, and a multi-electrode cuff around the hypoglossal nerve. While asleep the system selectively stimulates the hypoglossal nerve to protrude the tongue and pharyngeal dilators during inspiration, thereby enhancing airway patency. This therapy has a direct interplay with a patient's oral anatomy and therefore, dental factors can play a role in treatment efficacy. As more providers offer HGNS, dentists will be required to be knowledgeable to handle complications related to this treatment. We provide an example where dental intervention enhanced HGNS therapy.

Report of Case: A 75-year-old male presented to the sleep center with complaints of snoring, witnessed pauses in breathing during sleep, excessive daytime sleepiness, frequent movements during sleep, and morning nasal congestion. Subsequent sleep testing revealing OSA and the patient was initially treated with PAP and then deemed a candidate for HGNS. Following the HGNS procedure, the stimulation voltage was gradually titrated to $4.1 \mathrm{~V}$ with subjective benefits in OSA symptoms but noted bilateral pain on the lateral borders of the posterior tongue from the repetitive protrusions during sleep. He would frequently awaken at $4 \mathrm{AM}$ to pause the device as a result of his discomfort. A 2-night home sleep apnea test (HSAT) showed residual apnea with an AHI of $22.4 / \mathrm{hr}, \mathrm{SaO} 2$ nadir of $83 \%$ and $16.8 / \mathrm{hr}, \mathrm{SaO} 2$ nadir of $85 \%$ respectively. Higher HGNS stimulations voltage was needed but not tolerable as a result of the tongue irritation.

The patient was referred for dental assessment and multidisciplinary care to address his HGNS treatment complication for the patient's OSA. Patient had not been to the dentist in greater than 10 years and admitted to neglecting his teeth with little motivation to resume dental care. Intraoral examination revealed multiple large amalgam fillings on posteriors and broken \#18 DL and \#31 DL cusps resulting in sharp edges. After treatment discussion and informed consent, teeth \#18 and \#31 were adjusted, smoothed, and polished. Patient was referred back to sleep physician for continued care. On follow up, the patient stated improvement in the tongue pain from the HGNS and improvement in his sleep.

Discussion: As more patients are treated with HGNS, dentists and other healthcare providers will be required to address similar complications. The teeth and other structures within the oral cavity can prevent or limit the protrusion of the tongue. Dentists should be aware of this and other complications of HGNS. Additional cases will be presented to further highlight dental aspects of HGNS therapy. In addition, if the patient remains suboptimally treated with HGNS alone, dentists could potentially provide combination therapy (HGNS with oral appliance). Appliance selection needs to take into account clearance for tongue protrusion. Dentists have the opportunity to aid in the management of HGNS complications as part of a collaborative approach to sleep medicine.

\section{Support: None}

COI Disclosures: Dr. Simmons reports sitting on the advisory board and being a consultant for Inspire Medical Systems. 
ABSTRACT \#018

\section{TREATMENT OF SEVERE OBSTRUCTIVE SLEEP APNEA WITH ORAL APPLIANCE THERAPY.}

Dishti Goyal ${ }^{1}$, Walt Pfitzinger, DDS, MS $^{2}$ 1 Grinnell College, Grinnell IA; 2 Plaza Dental Group,
Columbia MO

Introduction: The American Academy of Sleep Medicine and American Academy of Dental Sleep Medicine recommend that oral appliance therapy (OAT) can be used as an alternative treatment option in patients with mild or moderate obstructive sleep apnea (OSA). For patients with severe sleep apnea, oral appliances can be considered if they have failed continuous positive airway pressure (CPAP).

Methods: We retrospectively analyzed 8 cases of severe OSA who failed CPAP and were treated successfully with an oral appliance, in our practice.

Results: There were 4 male and 4 female patients. The mean age was 53.5 years, mean body mass index (BMI) was 31 and mean Epworth sleepiness score was 9.8. All the patients had severe OSA with mean pretreatment apneahypopnea index (AHI) of 48.0 and mean SPO2 nadir of $85.3 \%$. All patients had an evaluation by a sleep physician and had failed CPAP therapy. These patients were fitted with a custom-made titratable oral appliance $\left(\right.$ SomnoMed $\left.^{\circledR}\right)$. Patients underwent a sleep study with the oral appliance, to determine its efficacy. In all cases the appliances required further titration from the initial setting either during or after the follow up sleep study. With OAT, posttreatment mean AHI was 4.2. These patients reported significant improvement in their symptoms. No significant adverse effects were reported.

Conclusions: Oral appliance therapy (OAT) can be an effective alternative treatment option in patients with severe obstructive sleep apnea who had failed CPAP therapy. However, the importance of a follow up sleep study for titration of the appliance is paramount to achieve maximum benefit.

\section{Support: None}

\section{COI Disclosures: None}

\section{LATE-BREAKING CASE REPORTS}

\section{CASE REPORT \#019}

\section{ORAL APPLIANCE CALIBRATION GUIDED BY OXIMETRY}

Walter da Silva Jr

\section{ABDSM International Certificant Instituto Walter Silva de Odontologia Bauru/Brazil}

Introduction: Oral appliance therapy (OAT) has been reached excellent outcomes. This process depends on a lot of steps, since OA choice, impression or scan, lab technical performance, calibration and a trustable check if $\mathrm{OA}$ is working well; beyond patient's information, objective data are also too important. Sleep dentists can ask for patient record his sleep by snore's app and use sleep time digital oximetry to follow the oxygen saturation during all sleep.

Report of Case: Male, 45 years, complains of huge sleepiness and snore (reported by his spouse). He was undergone to type 1 polysomnography which bring these data:

. AHI -21.7

. lowest oxygen saturation $-85 \%$

. ODI -22.2

Patient was not overweight and presented normal blood pressure. CPAP and OAT treatments were explained. Patient's preference was OAT.

A basal sleep time digital oximetry was done showing ODI=23 (similar to the found on basal sleep test).

A device with bilateral expanders and lateral and protrusive freedom was planned. After fit consultation (delivery in $50 \%$ of maximum protrusive motion), a new visit was scheduled after 7 days. Until there, a daily contact by messages or phone call was done asking if there were some complaints, doubts or pain. If necessary, an earlier consultation was booked.

In his first back to the office, he reported good sleep, less snore and somnolence. He was very excited and happy. It was done $1 \mathrm{~mm}$ of OA advancement, asked for new sleep time digital oximeter with OA and booked other consultation 2 weeks later.

The result of the new oximetry showed ODI=1, showing a great decrease in this important parameter. This outcome gave more trust indicating an OA therapeutic position. So, a new type 1 polysomnography was required with $\mathrm{OA}$. The data were: 
. AHI -4.3

. lowest oxygen saturation $-90 \%$

. ODI -3.9

Discussion: This case report showed a trustable way to observe if OAT is in going well, not only with patient and partners' report. Type 1 polysomnography is considered gold standard for diagnosis and follow up of obstructive sleep apnea. However, this test is expensive and few cities offer (in Brazil only big centers and some medium cities have). So, before refer patients to the second test (with OA), it's interesting that OA be on disease control position. A digital oximetry is easy, trustable and cheap tool that can be used. This device is also indicated for screening suspect patients.

Support: No financial support.

COI Disclosures: None.

\section{CASE REPORT \#020}

\section{COMBINATION THERAPY IN A PATIENT WITH SIGNIFICANT DENTAL COMPLICATIONS: A CASE REPORT}

Martin Denbar, DDS, D.ABDSM 1 , Aaron Glick DDS, D.ABDSM ${ }^{2}$

${ }^{1}$ Adjust Assistant Professor, Texas A\&M School of Medicine, Private Practice, Austin , Texas; ${ }^{2}$ Clinical Assistant Professor, Department of General Practice and Dental Public Health, The University of Texas Health Science Center at Houston School of Dentistry

Introduction: CPAP non-compliance with multiple comorbidities can be a challenge for medical providers to manage. Combination therapy (PAP therapy with oral appliance) can aid in managing a severe case of obstructive sleep apnea (OSA). However, a patient with poor oral dentition as the foundation for the oral appliance can provide a particularly complicated management strategy for medical and dental practitioner. This case will review the successful management of a severe OSA patient with damaged dentition, bilateral artificial temporal mandibular jaw (TMJ) joint replacement, and limited opening using combination therapy.

Report of Case: A 68-year-old female presented with uncontrolled hypertension, fibromyalgia, nasal allergies, severe sleep apnea, thyroid disorder, and severe claustrophobia. Patient had bilateral total TMJ joint replacement in 1980 with subsequent surgeries to remove left and right auricular fossa. In addition, patient has implant supported lower denture with maxillary missing teeth restored with $(1,2,5,7,9,15$, and 16). Remaining maxillary teeth are heavily restored and required anterior bridge replacement due to caries prior to fabrication of oral appliance. Patient had limited opening of $29 \mathrm{~mm}$ at initial visit. The patient was treated in coordination with her cardiologist and primary care provider.

Initial diagnosis of sleep apnea was AHI of 43.3 and nadir of $55 \%$. Due to claustrophobia, headgear discomfort, sleep interruption, and ineffectiveness of treatment patient was unable to tolerate CPAP and non-compliant with therapy.

Jaw stretching exercises were completed by the patient allowed increased vertical and horizontal movement. Upon completion patient was able to translate $3.25 \mathrm{~mm}$ past centric occlusion and comfortably wear the oral appliance (TAP 3). Patient had drastic improvement through treatment with oral appliance alone. Time above $90 \%$ $\mathrm{SpO} 2$ (T90) was $97.2 \%$ with elevated desaturation indices of 11.1 (4\% oxygen desaturation) and 23 (3\% oxygen desaturation). After completion of titration protocol and attachment of oral appliance to PAP nasal mask (Phillips Respironics DreamWear), patient showed an AHI of 2.8 with median pressures of $7.3 \mathrm{cmH}_{2} \mathrm{O}$ and time of usage at 5 hours and 29 minutes.

Discussion: The field of Oral Appliance Therapy goes far beyond the "simple" cases of mild/moderate sleep apnea. When patients present for treatment there needs to be available therapies that are cost effective and yet still comprehensive enough to offer the patient. Compromised dental management is a necessity in many cases in order to meet the patient's needs. This case is a classic example of the patient not having an ideal or adequate dental condition for treatment yet full management was obtained within the confines of basic dentistry with minimal cost and overall excellent results.

Support: None.

COI Disclosures: None. 